THÉMATA. Revista de Filosofía

No49, Enero-junio (2014) pp.: 203-215

ISSN: 0212-8365 e-ISSN: 2253-900X

doi: 10.12795/themata.2014.i49.11

\title{
DIGNIDAD Y BIOÉTICA: EL CASO DE LAS INTERVENCIONES BIOTECNOLÓGICAS DE MEJORA DE LA ESPECIE ${ }^{1}$
}

\author{
DIGNITY AND BIOETHICS: THE CASE OF THE HUMAN \\ ENHANCEMENT BIOTECHNOLOGICAL INTERVENTIONS
}

\author{
Francisco Javier López Frías ${ }^{2}$ \\ Universidad de Valencia (España)
}

Recibido: 19-04-2013

Aceptado: 30-06-2013

\begin{abstract}
Resumen: Este artículo trata de participar en el debate en torno al uso del concepto de dignidad en la bioética. No sólo tratará de mostrar que éste puede ser utilizado con sentido, sino que defenderá que se trata de un término clave necesario para la reflexión bioética, especialmente, dentro del debate sobre la mejora humana. Para ello, este concepto debe ser analizado al detalle y definido del modo más claro posible. Lo cual trataré de realizar de la mano de tres fuentes: la teoría del reconocimiento propuesta por Axel Honneth, la ética del discurso de Karl Otto Apel y Jürgen Habermas, y el enfoque de las capacidades de Martha Nussbaum y Amartya Sen-he de añadir una cuarta fuente a esta enumeración: la compuesta por el intento de armonizar estas tres fuentes realizado por los miembros del Departamento de Filosofía Moral de la Universidad de Valencia
\end{abstract}

Palabras-clave: bioética, dignidad, mejora humana, naturaleza humana

[1] Este estudio se inserta en el Proyecto de Investigación Científica y Desarrollo Tecnológico FFI2010-21639-C02-01, "Ética del discurso, política democrática y neuroética" financiado por el Ministerio de Ciencia e innovación y con Fondos FEDER de la Unión Europea. Becario de Investigación FPU (AP2009-4405) del Ministerio de Educación y Ciencia. Departamento de Filosofía del Derecho, Moral y Política. Universidad de Valencia.

[2] (Francisco.Javier.Lopez@uv.es) Becario FPU en el Departamento de Filosofía Moral de la Universidad de Valencia. Realiza su tesis sobre mejora humana y filosofía práctica del deporte bajo la dirección de Jesús Conill Sancho. Es miembro de diversas asociaciones filosóficas como la Asociación Internacional de Filosofía del Deporte (IAPS) y la Asociación Latina de Filosofía del Deporte (ALFiD). Ha realizado estancias de investigación en el Uehiro Center for Practical Ethics de la Universidad de Oxford, la Penn State University, y la Universidad de Roma "Foro itálico". 


\begin{abstract}
This paper shall take part in the debate on the use of the concept of dignity within bioethics. It shall attempt to show that such a concept belongs to bioethics and also that it is a key element for bioethics, especially, concerning issues related to human enhancement interventions. In order to do so, the dignity concept shall be clearly defined. In so doing, this article shall use three different sources: a) the theory of recognition sketched by Axel Honneth, b) Jürgen Habermas' and Karl O. Apel's discourse ethics; and c) the capabilities approach defended by Martha C. Nussbaum-a fourth source should be added: the proposal of the members of the Moral Philosophy Department of the University of Valencia which tries to combine the three aforementioned proposals in order to sketch a proper methodological approach to work within applied ethics. .
\end{abstract}

Key-words: bioethics, dignity, human enhancement, human nature.

\title{
1. La relevancia moral de las intervenciones biotecnológicas de mejora humana
}

El problema de la adecuación del concepto de dignidad al terreno la bioética no es nuevo ni, mucho menos, exclusivo del debate surgido en torno a la aplicación de las biotecnologías al ámbito médico. Es más, como advierte Leon R. Kass, ante las nuevas posibilidades y peligros que éstas presentan, los términos con que tradicionalmente hemos trabajado en bioética: o bien no funcionan, o requieren de una revisión a fondo. Así, sobre todo desde que la clonación humana es posible, el debate en torno a la dignidad ha resurgido con fuerza dentro de la bioética. Como respuesta al artículo de Ruth Macklin en el que defiende que la dignidad es un concepto inservible, el Comité propuso la realización de un informe, titulado Human Dignity and Bioethics: Essays Commissioned by the President's Council on Bioethics. Éste no sólo se enfrentaba uno de los problemas principales de la bioética actual: la mejora humana, sino que también mostraba que es necesario utilizar el concepto de dignidad para abordarlo.

Francis Fukuyama, Jürgen Habermas, Allen Buchanan, y Nicholas Agar, entre otros, han advertido que las técnicas de mejora humana pueden hacer peligrar el modo en que nos autorreconocemos como seres iguales y, en última instancia, dignos. Esta afirmación no parece tan alejada de la realidad si prestamos atención tanto a las bases morales que sustentan las relaciones humanas, como también a la historia de las mismas. Por ejemplo, Axel Honneth ha mostrado que la historia moral de la Humanidad ha avanzado a raíz de las diversas luchas que los marginados sociales han llevado a cabo en busca del reconocimiento de su dignidad: esclavos, miembros de los estamentos oprimidos, comunidades religiosas...

Tomando la distinción entre mejoras moderadas y mejoras radicales establecida por Nicholas Agar, defenderé que estas últimas generarían situaciones moralmente problemáticas al modo de los ejemplos históricos de discriminación nombrados con anterioridad. Así, por ejemplo, surgiría una raza posthumana de 
seres humanos mejorados y otra "normal", no-mejorada, cuyos intereses y naturaleza podrían ser tan opuestos entre sí que sería factible que emergieran serios problemas relativos al reconocimiento mutuo entre comunidades diferentes. Con el fin de aclarar este debate, algunos autores han tratado de encontrar ese "factor X”, en términos de Fukuyama, en virtud del cual estamos obligados a tratar a las personas como tal. Este "factor X" no es otra cosa que el concepto de dignidad. Por lo tanto, el debate en torno a la mejora humana requiere recurrir a ella.

\section{Utilidad del concepto dignidad}

Este apartado analizará la polémica en torno a la utilidad y necesidad del concepto de dignidad en el marco bioético. Discutirá directamente con aquellos que rechazan su uso para tratar de encontrarle un sitio en el discurso bioético actual. Como punto de partida refutaré la posición defendida por Ruth Macklin, que asegura que el concepto "dignidad"

«parece no tener significado más allá del que ya está inscrito en el principio ético médico del respeto por la persona [...] las apelaciones a la dignidad son, o bien re-elaboraciones de otras nociones más precisas, o simples eslóganes que no añaden nada a la comprensión del problema [Así pues, la] dignidad es un concepto inservible en la ética médica y puede ser eliminado sin que se pierda ningún contenidoß»».

Hay tres presupuestos a la base del artículo de Macklin. Primero, el concepto de respeto por la persona, formulado y defendido mayormente por la tradición kantiana, es más que suficiente para la reflexión bioética, pues cubre el mismo terreno que pretende el concepto de dignidad. Segundo, es preferible hablar de respeto por las personas porque se trata de un concepto mucho más claro que el de dignidad. De hecho, este último sólo ha aparecido en nuestros discursos morales a modo de herramienta política para enfrentarse a los horrores experimentados por la Humanidad en el S. xx. Por último, la dignidad es un concepto esencialmente religioso-suele entenderse que el hombre es merecedor de ella porque ha sido hecho a imagen y semejanza de Dios. Así, no se trata de un concepto válido para elaborar propuestas filosóficas laicas como las que persigue la bioética.

En resumen, el argumento que, a juicio de Macklin, muestra que el concepto de dignidad es inservible en bioética podría formularse del siguiente modo: a) no se ha ofrecido una definición clara del mismo, b) y sólo ha sido utilizado como herramienta con fines políticos desde mediados de S. Xx. Además, c) posee un trasfondo religioso que lo hace contrario a la naturaleza laica de los debates bioéticos. Por lo tanto, d) es mejor utilizar el término "respeto por las personas". El cual sí está definido claramente por la tradición kantiana, posee más historia que el concepto de dignidad, y encaja con la naturaleza laica de los problemas bioéticos. Este artículo afirma que estas cuatro afirmaciones son incorrectas.

[3] R. Macklin: "Dignity is a useless concept”, p. 1419 en BMJ, 327, 2003, pp. 1419-1420.

THÉMATA. Revista de Filosofía, Nº49 enero-junio (2014) pp.: 203-215

doi: 10.12795/themata.2014.i49.11 


\subsection{La dignidad ¿"se dice de muchas maneras"?}

Afirma Martha C. Nussbaum que el concepto de dignidad es «una noción intuitiva que no está de ninguna manera completamente clara ${ }^{4} »$. Otros autores como Holmes Rolston III y Leon R. Kass coinciden con ella al respecto. No obstante, ninguno de ellos afirma que este concepto sea inservible e innecesario para la bioética, tal y como hace Ruth Macklin. Es cierto que el concepto de dignidad ha sido utilizado en sentidos muy diversos. Por ejemplo, Leon R. Kass distingue entre dos sentidos. Por un lado, la dignidad básica de ser humano, por otro, la dignidad completa de ser un ser humano. En el primer caso hablamos de un uso de mínimos, es decir, referido a aquello que todos los seres humanos comparten por el mero hecho de ser un ser humano. Por el contrario, el segundo caso es de máximos, entendiendo por ello que no es algo que todas las personas compartan de forma inicial, sino que surge como fruto de sus acciones. Por su parte, Nick Bostrom también diferencia entre un concepto reducido de dignidad y otro amplio. El primero atribuye dignidad a algo porque tiene un valor intrínseco. Por otro lado, el sentido amplio de la dignidad se fundamenta en el reconocimiento que los otros hacen de algo. A diferencia de Kass, Bostrom defiende que ambos conceptos de dignidad—que no se restringe a los seres humanos ${ }^{5}$ - poseen un carácter gradual.

Ante tal variedad de formas de conceptualizar la dignidad, podemos afirmar que: si bien la diversidad a la que se refiere Macklin con su argumento es cierta. Sin embargo, sus conclusiones al respecto son erróneas porque: primero, la ambivalencia que presenta el concepto de dignidad no implica que éste no sea útil ni necesario para la bioética. Como afirma el título de este apartado, puede ser que la dignidad, parafraseando a Aristóteles, "se diga de muchas maneras". Segundo, a pesar de esta multiplicidad de sentidos en que se entiende este concepto, puede ser que exista una definición de mínimos que englobe a todos éstos.

Este artículo defenderá que no hay diversos conceptos de dignidad, sino que existen diversos planos en los que ésta se percibe. Es decir, ofrece una versión mixta entre los dos conceptos de dignidad propuestos por Bostrom y Kaas. A la vez que reconoce que los sujetos merecedores de dignidad tienen algo en sí que debe protegerse - plano normativo. Sin embargo, esto no les otorga directamente dignidad, sino que ésta debe reconocerse y protegerse intersubjetivamente-plano del reconocimiento. Así, la dignidad, entendida como eso que todos comparti-

[4] M. Nussbaum: Creating Capabilities: The human developmental approach, Massachusetts, The Belknap Press of Harvard University Press, 2011, p. 29.

[5] Así por ejemplo, Nick Bostrom afirma que incluso un muñeco de nieve tiene características que le hacen merecedor de cierta dignidad en sentido reducido, pues son apreciadas por los seres humanos de un modo especial, al igual que, por ejemplo, podría serlo un bonito paisaje natural o una especie animal en particular. "consideremos el largo, lento, y triste ocaso de un muñeco de nieve que se está fundiendo [...] ¿No reconocería un observador idealmente sensible cierto grado de dignidad como cualidad en el bueno del muñeco de nieve?» N. Bostrom: "Dignity and Enhancement", p. 200, PREsident's COUnCIL ON BIOETHICs, cit., pp. 173-206.

THÉMATA. Revista de Filosofía, Nº49 enero-junio (2014) pp.: 203-215 doi: 10.12795/themata.2014.i49.11 
mos y que debe ser reconocido, no acepta grados. Lo único que puede concebirse como gradual es el proceso por el cual eso que merece protección va saliendo a la luz e institucionalizándose a través de la acción de las comunidades políticas.

En su obra acerca de la idea de Europa y su fundamentación moral, Habermas dedica un capítulo completo a la idea de dignidad y a cómo ésta ha ido ligada a la evolución moral de la Humanidad: "El concepto de dignidad humana y la utopía realista de los derechos humanos". En este capítulo, reconoce a los detractores del concepto de dignidad que éste

"sólo alcanzó a materializarse después de la Segunda Guerra Mundial en los textos del derecho internacional y en las constituciones nacionales vigentes desde entonces [, con una] excepción a mediados de siglo XIX [...] los debates para la abolición de la pena de muerte y del castigo corporal en la Constitución de Fráncfort de marzo de $1849^{6}$ ».

No obstante, aclara que el hecho de que el concepto de dignidad no haya entrado a formar parte importante del vocabulario jurídico hasta tan tarde, no excluye que sí lo haya hecho en el filosófico-autores como los estoicos o Kant trataron sistemáticamente la dignidad. Es más, añade Habermas, el concepto de dignidad constituye una fuente moral que ha estado presente a lo largo de toda la Historia, de hecho, es aquella de la que beben todos los derechos fundamentales:

«La experiencia de las violaciones de la dignidad humana tiene una función reveladora: ya sea en vista de condiciones de vida sociales insoportables y la marginación de clases sociales empobrecidas; ya en vista del trato desigual a hombre y mujeres en el lugar de trabajo, o de la discriminación de extranjeros y de minorías culturales, lingüísticas, religiosas o raciales; [...] A través de este proceso, la intuición subyacente penetra primero en la conciencia de los afectados y después en los textos legales, donde encuentra su articulación conceptual"».

Así pues, no es cierta la afirmación de Macklin de que la dignidad no tuvo un peso histórico relevante hasta que la Humanidad presenció los horrores cometidos a principios de S. Xx. Del mismo modo, tampoco es cierto que este concepto tenga una naturaleza única y exclusivamente religiosa, todo lo contrario, éste tiene su fundamento en un hecho tan básico como la vulneración de aquellas condiciones que los individuos han considerado desde siempre que les son debidas por el mero hecho de ser lo que son: seres humanos.

\subsection{Más allá del respeto por la persona}

Ya se ha respondido a los tres primeros puntos en los que Macklin basaba su argumento: a) la multiplicidad del concepto "dignidad", b) el uso político del mismo y c) su esencia religiosa. Sin embargo, queda aquél con el que parece más

[6] J. Habermas: La constitución de Europa, Trotta, Madrid, 2012, pp. 14-15.

[7] Ibíd., p. 16.

[8] Ibíd., p. 18.

THÉMATA. Revista de Filosofía, Nº 49 enero-junio (2014) pp.: 203-215 doi: 10.12795/themata.2014.i49.11 
difícil enfrentarse, a saber, el que afirma que la dignidad no significa nada distinto a la idea de respeto por la persona. Así pues, se están "multiplicando entes sin necesidad", parafraseando a Guillermo de Ockham, dando un nombre nuevo a algo que, de hecho, ya funciona desde antaño. Puede ser que los tres primeros argumentos de Macklin sean falsos, pero que, sin embargo, este último mantenga su validez. Así, podemos estar en lo cierto afirmando que la procedencia del término dignidad es más antigua de lo que sus detractores afirman, así como en que el concepto puede entenderse en diversos modos que apelan a uno central, pero Macklin puede tener razón en que este sentido central es el del respeto a la persona. Por lo tanto, no existe motivo alguno para hablar de dignidad, pues ya disponemos de un término que ha ocupado exitosamente su lugar con anterioridad?.

Sin embargo, Macklin no está en lo cierto. La dignidad no puede ser igualada a la idea del respeto por la persona, al menos en el sentido en que ella entiende esta última: como respeto por la autonomía de los sujetos. El término "dignidad" abarca y significa mucho más que el de "respeto por la persona". Tal y como afirma Daniel Davies en su crítica a la noción de respeto por la persona empleada por Ruth Macklin, ésta es entendida a la luz de dos convicciones éticas: a) los individuos deben ser tratados como agentes autónomos, y b) las personas con autonomía reducida deben ser protegidas. De este modo Macklin toma los conceptos de respeto por la persona y respecto por la autonomía como iguales. Respetar significa: "abstenerse de interferir la elección libre y sin restricción de un sujeto autónomo. ${ }^{10}$

Siguiendo esta línea interpretativa, Richard J. Neuhaus reprocha a Macklin que si bien el respeto a la autonomía es uno de los principios éticos fundamentales de la bioética, sin embargo, éste no es suficiente. De ahí que muchos autores hayan defendido la existencia de cuatro principios fundamentales de la bioética, entre ellos Thomas L. Beauchamp y James F. Childress, o Diego Gracia en el ámbito español. En este sentido, Neuhaus destaca la importancia del "no dañar", es decir, del principio de no maleficencia, que no es reducible a la preocupación por la autonomía, pues nos invita a proteger y mantener aquello que es reconocido como bueno en nuestro ser - de hecho, Diego Gracia incluye el principio de beneficencia como la otra cara de la moneda de la que el principio de no maleficencia es parte.

Sin embargo, esta crítica necesita de algo más para ser efectiva; si fuera posible igualar eso que Neuhaus denomina "lo que es bueno en nuestro ser" con la autonomía, entonces podríamos afirmar que el concepto de respeto por la persona cumple la misma función que el de dignidad, y Macklin tendría razón de nuevo. Sin embargo, no parece que Neuhaus dé un significado tan reducido a eso que destaca como "lo valioso del ser humano", pues el texto se refiere constantemente a "la protección de aquellos que tienen más posibilidad de ver su dignidad violada", es

[9] Como mostró, por ejemplo, el Informe Belmont, que se construyó a raíz de tres principios: respeto por la persona, beneficencia, y justicia.

[10] D. Davies, "Human Dignity and Respect for Persons: A Historical Perspective on Public Bioethics", p. 27 en The President's Council on Bioethics, cit., pp. 19-38.

THÉMATA. Revista de Filosofía, N 49 enero-junio (2014) pp.: 203-215

doi: 10.12795/themata.2014.i49.11 
decir, a los más desfavorecidos y desprotegidos. No se trata aquí, pues, del establecimiento de fronteras de la naturaleza humana que no pueden ser cruzadas, sino del empoderamiento de aquellos que ven sus capacidades truncadas.

El lenguaje de la dignidad no está asociado exclusivamente al modo de tratar y proteger a los individuos en función de su autonomía, sino que se refiere a algo más: tener la capacidad de preservar una vida característicamente humana. Siguiendo la propuesta del enfoque de las capacidades, la pregunta central de la dignidad se refiere a "aquello que cada persona es capaz de hacer y $\operatorname{ser}^{11}$ ». Esto muestra una conceptuación más positiva del término "dignidad" que no se limita a la negatividad del impedir dañar, o a la autonomía, sino que se preocupa por aquello que las personas son y, sobre todo, por el hecho de que, en verdad, lleguen a alcanzar esos modos de vida que tienen razones para valorar. ¿Puede reducirse esta forma de comprender la dignidad al respeto de la autonomía de las personas?

\section{Dignidad humana, naturaleza, y normatividad}

\subsection{Dignidad ¿de la especie? ¿La hay más allá de ella?}

El apartado anterior ha sugerido que la dignidad recibe su contenido de la concepción normativa de la naturaleza humana que alberguemos. Ésta no es un simple hecho empírico, que está ahí para que lo estudiemos, modifiquemos, y manejemos, sino que posee un significado moral. Por ello, parece necesario que una investigación sobre la dignidad esté referida al concepto de naturaleza humana. Hay dos propuestas principales sobre qué es aquello que confiere normatividad a la naturaleza humana. Una es la concepción especista, que afirma que aquellas características que nos hacen formar parte de la especie poseen un valor intrínsecamente normativo. Por el contrario, la concepción anti-especista afirma que esta normatividad no surge del simple hecho de pertenecer a la especie sino de poseer unas características determinadas.

La gran diferencia entre estas dos corrientes radica en el trato hacia aquellas personas que siendo claramente humanas, no se sabe cómo concebirlas desde un punto normativo porque no ejercen de pleno las funciones más típicamente humanas. Por ejemplo, los enfermos mentales, los discapacitados psíquicos, o los niños, no son poseedores de un juicio racional, sin embargo, son concebidos como seres humanos porque tienen la capacidad de desarrollarlo — bien sea con el paso del tiempo, como los niños, o por medio de intervenciones médico-terapéuticas, como en el caso de los dos primeros — o porque comparten una fisiología común con el resto de seres humanos. Mientras que para los defensores de la definición especista estos tres tipos de personas serían dignos, por el contrario, para el no-especista esto resultaría problemático de aceptar, pues dependería de la cualidad en base a la que la dignidad es conferida. Por ejemplo, si se fundamenta en la capacidad racional de los sujetos, ninguno de los tres tipos de personas mencionadas tienen pleno uso de razón.

[11] G. Meilaender, "Human Dignity: Exploring and Explicating the Council's Vision", p. 258 en The President's Council on Bioethics, cit., pp. 253-277. El énfasis que aporta la cursiva es mío.

THÉMATA. Revista de Filosofía, No 49 enero-junio (2014) pp.: 203-215

doi: 10.12795/themata.2014.i49.11 
Este artículo defenderá una propuesta "mixta" entre ambas. Para ello se basará en tres fuentes. Primero, la antroponomía kantiana. Segundo, la concepción de la dignidad y la naturaleza humana ofrecida por Nussbaum en su versión del enfoque de las capacidades. Tercero, la hermenéutica crítica propuesta por la ética discursiva de Habermas. Con ello, propondrá una concepción de dignidad que se cimenta no sólo en la capacidad racional de los sujetos, sino en diversas potencialidades propias que capacitan al sujeto para actuar moralmente en los términos que explicita la ética del discurso.

Cualquier ser que encarne esas capacidades deberá ser considerado digno. Hasta ahora, sólo los seres humanos son candidatos a ello. Sin embargo, los avances biomédicos de mejora humana prometen que seres diseñados artificialmente, como los híbridos, los androides, las quimeras... también poseerán estas capacidades. De este modo, compartirán una sustancia normativa con los seres humanos que nos obligará a considerarles como merecedores de dignidad. Por tanto, a modo de resumen, este artículo propondrá el uso de unos criterios normativos antroponómicos que, aunque extraídos de la especificidad de los seres humanos, pueden ser aplicados a futuras especies. ¿Cuáles son estas capacidades o potencialidades propias de la especie humana que les convierte en seres morales y, por extensión, en seres merecedores de un reconocimiento digno? ¿Cuál es esa sustancialidad que confiera normatividad a su naturaleza?

\subsection{Esbozo de una antroponomía ${ }^{12}$ ¿posthumana?}

Apel definió a las personas ${ }^{13}$ como "todos los seres capaces de comunicación lingüística que son reconocidos en todas sus acciones y expresiones como interlocutores virtuales"14. Según Adela Cortina, siguiendo con la propuesta de corte intersubjetivo de la ética del discurso, una persona es «quien tiene las

[12] El término "antroponomía" hace referencia a la pretensión kantiana expuesta en $L a$ metafísica de las costumbres de elaborar una antropología moral como complemento de una metafísica de las costumbres. Esta antroponomía sería la aplicación de lo expuesto en la metafísica y, por ello, consistiría en la exposición de «las condiciones subjetivas, tanto obstaculizadoras como favorecedoras, de la realización de las leyes de la primera [la metafísica de las costumbres] en la naturaleza humana, la creación, difusión y consolidación de los principios morales» I. Kant, Metafísica de las costumbres, trad. por Adela Cortina y Jesús Conill, Madrid, Tecnos, 2008, p. 21. Esta antropología fue elaborada por Kant en uno de sus últimos escritos: Antropología en sentido pragmático. Como ha afirmado Jesús Conill, esta obra viene a cerrar la propuesta eleuteronómica kantiana, que muestra que la auténtica humanidad radica en la moralidad, es decir, en la capacidad que los individuos poseen para autolegislarse. Así, «la antropología constituye la parte empírica de la filosofía moral kantiana». J. Conill, "Eleuteronomía y antroponomía en la filosofía práctica de Kant”, p. 274 en Julián Carvajal Cordón (ED.), Moral, derecho y política en Immanuel Kant, Ediciones de la Universidad de Castilla-La Mancha, Cuenca, 1999, pp. 265-284.

[13] K. O. Apel, "El a priori de la comunidad de comunicación y los fundamentos de la ética" (1967), en La transformación de la filosofía, Vol. 2, Madrid, Taurus, 1985, p. 380, cit. por J.C. Siurana, La idea de sujeto en la ética del discurso de Karl-Otto Apel aplicada al problema de las directrices anticipadas en el ámbito de la ética biomédica (Valencia), Tesis dirigida por Adela Cortina, 2005, p. 49.

[14] J.C. Siurana, La idea de sujeto en la ética del discurso de Karl-Otto Apel aplicada al problema de las directrices anticipadas en el ámbito de la ética biomédica, cit. p. 91.

THÉMATA. Revista de Filosofía, N 49 enero-junio (2014) pp.: 203-215 doi: 10.12795/themata.2014.i49.11 
capacidades requeridas para la autoconciencia, para el mutuo reconocimiento de la dignidad, para actuar desde la libertad y asumir su responsabilidad ${ }^{15}$. Es decir, son humanos aquellos que poseen las capacidades necesarias para la comunicación lingüística, sólo a través de ésta, los seres humanos podemos conformar una comunidad moral verdadera que

«está formada por sujetos capaces de comunicación [...] capaces de llevar adelante la vida juntos, lo que significa la capacidad de decidir conjuntamente las normas que favorecerán el respeto mutuo y el empoderamiento de los débiles, la capacidad de cumplir deberes, emprender proyectos comunes a través de la deliberación, la negociación y el compromiso; la capacidad de pensar qué leyes serían más justas para su convivencia y para el cuidado de los otros seres ${ }^{16}$ ».

El ser humano, en tanto que poseedor del lenguaje, ha mostrado ciertas capacidades que urge reforzar y que deben protegerse. Éstas son la base normativa para que se reconozca su dignidad. Dichas capacidades son la base evaluativa mediante la que establecemos aquello que nos parece irrenunciable para llevar una vida digna, o más bien, "el tipo de vida que cada uno tiene razones para valorar" 17 . Por lo tanto, éstas no son algo que está ahí de forma natural sin más, confiriéndonos un valor cuasi-divino, sino que deben sacarse a la luz, reconocerse, y potenciarse. Así, la dignidad sólo emerge dentro de las comunidades morales humanas a raíz del reconocimiento que otros hacen de las siguientes capacidades ${ }^{18}$ :

- Autoconciencia.

- Actuar desde la libertad en tanto que se responsivo.

- Ser responsable de estas acciones.

- Ser capaz de reconocer estas mismas capacidades en los otros.

Ninguno de estos caracteres hace mención a una fisiología común o al cuerpo humano como tal, sino a capacidades que brotan de la especificidad humana. Este criterio es contrario al que toma, por ejemplo, Nicholas Agar al establecer la distinción entre mejoras moderadas y mejoras radicales. A su juicio, las primeras son aquellas que mejoran biotecnológicamente a los individuos hasta límites y realidades que alguna vez han sido alcanzadas por el ser humano-por ejemplo, le parece que no hay ningún reproche moral, de inicio, en generar sujetos con un coeficiente intelectual igual al de Stephen Hawkins. Por contra, Agar se opone a las mejoras radicales cuya puesta en práctica daría lugar a seres que nunca hemos conocido y que serían difícilmente reconocibles según los cánones de lo humano que hoy manejamos-por ejemplo, personas

[15] A. Cortina, Las fronteras de la persona, cit., p. 185.

[16] Ibid, p. 187.

[17] A. Sen, Desarrollo y libertad, Editorial Planeta, Barcelona, 2000.

[18] A. Cortina, Las fronteras de la persona, cit., p. 187.

THÉMATA. Revista de Filosofía, Nº 49 enero-junio (2014) pp.: 203-215 doi: 10.12795/themata.2014.i49.11 
con cerebros robóticos cuya capacidad de comunicación y razonamiento fuera completamente distinta de lo conocido hasta el día de hoy.

Si actualmente no existen seres que posean una configuración fisiológica completamente distinta a la especie humana pero sean capaces de autoconciencia, responsividad, responsabilidad, y reconocimiento. Sin embargo, las biotecnologías aventuran que esta posibilidad será factible dentro de unos años, cuando la creación de seres híbridos, quimeras, o androides será una realidad en vez de una cuestión de ciencia ficción; ¿no pueden estos seres creados, que no se incluirían dentro de la especie humana, ser a su vez considerados como seres dignos si cumplen con estos cuatro criterios? Y, por lo tanto, ¿no hablaríamos en tal caso de una antroponomía "posthumana"-aunque parezca paradójico hablar de algo que es "antropo-" y a la vez posthumano?

\subsection{Limites del posthumanismo: una propuesta de mínimos}

Este apartado ofrecerá una concepción de mínimos del término dignidad y, tomando la distinción establecida por Agar, afirmará que serán mejoras moderadas y aceptables aquellas que no trastoquen aquel fondo normativo sobre el que se basa la adscripción de la dignidad de los sujetos. Por contra, llamaré mejoras radicales a aquellas que generen sujetos que no puedan desarrollar alguna de aquellas cuatro capacidades. Así, la propuesta de este trabajo es que los límites del posthumanismo están en los rasgos morales definitorios que nos permiten formar parte de una misma comunidad moral. Mientras el posthumano pueda seguir siendo miembro de ella, las intervenciones biotecnológica estarán, de inicio, aceptadas, pues se puede decir que, aunque el sujeto posea una fisiología posthumana, puede comportarse como un ser humano desde el punto de vista normativo. Esta es también la posición tomada por Allen Buchanan, quien discutiendo en torno al problema de la condición moral de los individuos intervenidos con vistas a ser mejorados afirma:

«[P]odría ser cierto que nosotros los seres humanos tuviéramos ciertos derechos en virtud de nuestra humanidad, pero podría ser también cierto que hay otros seres-por ejemplo, formas de vida inteligente no humana en otros planetas-que también los poseen [...] lo que importa desde el punto de vista de la concesión de derechos humanos es que un individuo posea ciertas capacidades o intereses; el hecho de que otros individuos los posean en un grado mayor es irrelevante ${ }^{19}$ ».

En este momento es cuando debe mencionarse el argumento habermasiano en contra de las intervenciones biotecnológicas de mejora humana. Dado que las comunidades políticas surgen como ese envoltorio institucional que protege la comunidad moral de los seres capaces de autocomprensión, responsividad, responsabilidad, y reconocimiento, el socavamiento de esta base

[19] A. Buchanan, Beyond Humanity?..., cit., pp. 214-215.

THÉMATA. Revista de Filosofía, Nº49 enero-junio (2014) pp.: 203-215 doi: 10.12795/themata.2014.i49.11 
normativa-antroponómica-tendría consecuencias nefastas para nuestras sociedades democrático-liberales actuales. Desde el momento en que hay sujetos que no pueden pertenecer de pleno a la comunidad moral que ésta pretende proteger, las comunidades políticas pierden su sentido ${ }^{20}$ —en tal caso no tendría sentido hablar de mejora, sino de "peora".

\section{Conclusión: Una dignidad de mínimos como criterio normativo para las intervenciones de mejora humana}

Este artículo partió del desafío planteado por Macklin al mundo de la filosofía moral, según el cual, el concepto de dignidad es inservible. Vimos que sus razones para defender esta posición eran tres: a) basta con el principio del "respeto por la persona" para resolver los problemas que se tratar de abordar utilizando el concepto de dignidad; b) es más, éste es un término reciente, cuya aparición en el mundo de la filosofía moral se ha debido a los intentos de concienciación en contra de los horrores vividos por la Humanidad a principios del S.xx; por último, c) la polémica posición de Macklin añade que no existe, ni existió una definición clara de la dignidad, así pues, no se puede trabajar con ella desde un punto de vista teórico.

Para desmontar las premisas en que se sustenta la crítica de Macklin, se defendió que aunque pueda ser cierto que el concepto de dignidad se haya utilizado en diversos planos, esto no significa que sea inservible. Pues, utilizando las reflexiones aristotélicas sobre los diversos sentidos del ser, puede ser que estos sentidos se refieran a uno central. Dicho elemento central es lo que se ha tratado de captar aquí a través de una definición de mínimos de la dignidad, que no es otra cosa que aquellas características que convierten a los sujetos en agentes morales, las cuales deben ser protegidas y reconocidas intersubjetivamente.

Siguiendo la propuesta de Adela Cortina, defendí que las cuatro capacidades que dan sentido a esta concepción de mínimos de la dignidad son: la autoconciencia, la responsividad, la responsabilidad, y el reconocimiento del otro. Así pues, defendimos que cuando una intervención biotecnológica pone en peligro la posesión de una de estas capacidades hablaríamos de mejora radical—o "peora" - y la consideraríamos como moralmente rechazable. En contraposición a aquellas intervenciones, que denominé como "moderadas", que sí deberían aceptarse pues no trastocan ese fondo de normatividad que hace a los seres merecedores de dignidad.

[20] Por ello, David DeGrazia, Allen Buchanan y otros desarrollaron un debate en el volumen 38 del Journal Medical of Ethics en torno a lo posthumano y los derechos, en el que analizaron si los posthumanos, en tanto que podrían no encajar en las comunidades morales humanas, merecerían una consideración política y legal especial, o si por el contrario se les podría considerar como miembros de una misma comunidad moral y política.

THÉMATA. Revista de Filosofía, Nº 49 enero-junio (2014) pp.: 203-215 doi: 10.12795/themata.2014.i49.11 
Este trabajo ha mostrado que esta definición de mínimos es esencial en bioética, así como en la Historia del desarrollo moral de los sujetos. Una vez defendido esto, este texto se propone la tarea de saber si, en relación al debate en torno a la mejora humana, esta definición de mínimos es sólo aplicable a una especie en concreto: la humana. Para ello, se defiende una concepción mixta entre los especistas y los no-especistas. Pues si bien se parte de una antroponomía del estilo a la kantiana, que trata de determinar cuáles son aquellas capacidades que nos posibilitan actuar moralmente, sin embargo, estas capacidades se hacen extensivas a todas las especies que puedan poseerlas. De modo que se puede hablar de una "antroponomía posthumana". 


\section{Referencias bibliográficas:}

Agar, A., Liberal Eugenics: in defence of human enhancement, Oxford, Blackwell Publishing, 2004.

Agar, A., Humanity's End: Why We should Reject Radical Enhancement, Cambridge: MA, MIT Press, 2010.

Bayertz, K., GenEthics: Technological Intervention In Human Reproduction as a Philosophical Problem, Cambridge, Cambridge University Press, 1994

Buchanan, A., Beyond Humanity?: The Ethics of Biomedical Enhancement, Oxford University Press, Oxford, 2010.

Conill, J. El enigma del animal fantástico, Madrid, Tecnos, 1991.

Cortina, A., Ética sin moral, Madrid, Tecnos, 2000

Cortina, A., Neuroética y Neuropolítica: Sugerencias para una educación moral, Madrid, Tecnos, 2011,

Cortina, A., García-Marzá, D. (Eds.), Razón pública y éticas aplicadas: los caminos de la razón práctica en una sociedad pluralista, Madrid, Tecnos, 2003. Consejo de Bioética del Presidente de los Estados Unidos, Human Dignity and Bioethics, Washington, 2008.

Fukuyama, F., El fin del hombre: consecuencias de la revolución biotecnológica, Madrid, Punto de Lectura, 2003.

Habermas, J., El futuro de la naturaleza humana, El futuro de la naturaleza humana, Barcelona, Paidós, 2009, p.33.

Harris J., Enhancing Evolution: the ethical case for making better people, Princeton, N.J.; Princenton University Press, 2007.

Miller, P. \& Wilsdon, J., Better Humans? The politics of human enhancement and life extension, London, Demos, 2006.

Pols, A.J.K \& Houkes, W., "What is morally salient about enhancement technologies?" en Journal of medical ethics, 37 (2), 2011, pp. 84-87.

Rifkin, J., The Biotech Century: How Genetic Commerce Will Change de World, London, Penguin Putnam, 1998.

Ryberg, J., Petersen, Th., Wolf, C., (Eds.), New Waves in Applied Ethics, London, Pelgrave Macmillan, 2008.

Savulescu, J. \& Bostrom, N., Human Enhancement, Oxford, Oxford University Press, 2007

Savulescu, J., Meulen, R. ter, Kahane, G., Enhancing human capacities, Oxford, Wiley-Blackwell, 2011.

Walton, D., "Plausible Deniability and Evasion of Burden of Proof", Argumentation, 10, 1996, pp. 47-48. 
